\title{
RADIOGRAFÍA DE LA BRECHA DIGITAL EN CHILE: ¿SE JUSTIFICA LA INTERVENCIÓN DEL ESTADO?*
}

\section{Claudio A. Agostini y Manuel Willington}

En las últimas décadas la aceleración del desarrollo de las tecnologías de información y comunicación —en particular de Internet y el acceso a través de conexiones de banda ancha- ha generado transformaciones importantes en las sociedades. Sin embargo, especialmente en los países subdesarrollados y en desarrollo, el acceso a estas tecnologías y a sus beneficios no es parejo y hay diferencias significativas entre distintos grupos de la población. Esta llamada "brecha digital" ha llevado a muchos países, y Chile no ha sido la excepción, a preocuparse de promover el acceso a estas tecnologías. Así, hace ya varios años se crearon programas de infocentros en diversas comunas y se estableció la red Enlaces en las escuelas públicas. En los últimos años, sin embargo, la discusión en torno al fomento del acceso se ha centrado en el diseño de un subsidio a la demanda por conexión de banda ancha a nivel del hogar, política que lleva implícita el supuesto de que el problema central es el precio del acceso al servicio.

La radiografía de la brecha digital que realizamos en este trabajo persigue tres objetivos. Primero, caracterizar la evo-

Claudio A. Agostini. Doctor en Economía, University of Michigan. Profesor Asociado, ILADES-Universidad Alberto Hurtado. Email: agostini@uahurtado.cl.

Manuel Willington. Doctor en Economía, University of Pennsylvania. Profesor Asistente ILADES-Universidad Alberto Hurtado. Email: mwilling@uahurtado.cl.

* Agradecemos a María José Schaeffer por su valioso apoyo en la investigación. 
lución de esta brecha entre 2006 y 2009 en sus dimensiones "clásicas": la tenencia de PC y la conexión de banda ancha en el hogar. Segundo, caracterizarla en otro aspecto potencialmente más relevante que el acceso, como es el uso de Internet, variable sobre la cual se analizan aspectos complementarios como la frecuencia de uso, el lugar de uso y los tipos de uso. El tercer objetivo es ilustrar, a partir del análisis de las razones principales que la gente declara para no usar Internet o no conectarse, la importancia de otros factores que, más allá del precio de la conexión, invitan a analizar diferentes opciones de políticas.

Palabras clave: brecha digital; banda ancha; Internet; políticas de fomento.

Clasificación JEL: L86, L96, O33, O38.

\section{Introducción}

E

avance de las tecnologías de la información y comunicaciones en las últimas décadas ha generado una transformación importante en diversos sectores de la economía, más allá de las comunicaciones. En particular, a partir de la introducción y masificación de Internet en los últimos quince años se han producido transformaciones en las relaciones laborales con el teletrabajo, en las relaciones entre los ciudadanos y el Estado con el desarrollo del gobierno electrónico, en la educación por medio del e-learning, en el comercio por el surgimiento del comercio electrónico e incluso en la política a partir de los nuevos canales de comunicación.

El impacto económico y social del uso de Internet ha sido de tal magnitud que muchos países han considerado necesario fomentar su acceso y uso a través de políticas públicas específicas. Es así como existen para estos efectos créditos tributarios al ingreso (Suecia y Dinamarca), subsidios a la demanda (Italia), subsidios a la oferta (Corea del Sur), reducciones en el impuesto a las ventas (IVA), creación de centros comunitarios en el estado de Kentucky y subsidios a la compra de computadores (Kentucky, Tennessee). Adicionalmente, distintos programas tienen distintos públicos objetivo: hogares (Suecia), escuelas (Estados 
Unidos), pequeñas empresas (Escocia y el estado de Ohio), estudiantes (Nueva York) e incluso etnias (Estados Unidos).

Chile no es la excepción en este camino y durante los últimos años se han considerado políticas públicas que conllevan un rol más activo del Estado para aumentar la penetración de la banda ancha en el país. De hecho, ya se han destinado recursos públicos para incrementar el acceso a Internet en lugares públicos, a través de la creación de infocentros en distintas comunas y de la red Enlaces en las escuelas públicas. Adicionalmente, la autoridad de telecomunicaciones ha propuesto en múltiples oportunidades la implementación de un subsidio a la demanda que permita que un mayor número de personas tenga acceso a Internet con banda ancha en el hogar, en especial los hogares de menores ingresos.

Sin duda, hay externalidades positivas asociadas a Internet que justificarían la intervención del Estado. Más allá de las tradicionales externalidades de red (por ejemplo, el que haya un miembro adicional en la red beneficia no sólo a ese miembro sino a todos los demás), el acceso a Internet a través de banda ancha permite la difusión de ideas e información en mercados en los cuales crecientemente la información es un insumo relevante, lo cual facilitaría el desarrollo y adopción de innovaciones. La infraestructura de banda ancha junto a tecnologías de información puede entonces aumentar la productividad en la economía. Sin embargo, ello depende de cómo y para qué se usen las tecnologías de información y la presencia de otros insumos complementarios como el trabajo calificado (Autor et al., 2003). Por ello es que considerar el uso que se da a Internet es un aspecto relevante, ya que las externalidades positivas asociadas a Internet dependen en gran medida de las aplicaciones que se hagan (por ejemplo, la búsqueda de información tiene efectos positivos, mientras que la descarga ilegal de música no).

La magnitud de estas externalidades puede ser importante y si bien no hay estimaciones al respecto en la literatura económica, los resultados de Czernich et al. (2009) usando datos para 25 países de la OECD muestran que un aumento de 10 puntos porcentuales en la tasa de penetración de banda ancha aumentaría el crecimiento anual del PIB per cápita entre 0,9 y 1,5 puntos porcentuales.

En Chile, de acuerdo a la encuesta CASEN la penetración de Internet (usuarios de seis años de edad y más) pasó de 18,4\% en 2000 a $27,8 \%$ en 2003 y a $40,2 \%$ en 2006 . Si bien este nivel de penetración es 
alto para Latinoamérica y un poco más bajo para el promedio de países de la OECD (50\% en 2005), hay diferencias importantes entre distintos sectores de la población. Por ejemplo, la penetración en el decil de más altos ingresos es de 70\%, mientras que en el de menores ingresos es $14 \%$. La diferencia es aún mayor por nivel educacional: en los adultos con educación superior la tasa es de $61 \%$ y en los que sólo tienen educación básica es de $1 \%$. La existencia de estas brechas en la penetración de Internet es lo que ha llevado a la Subsecretaría de Telecomunicaciones a plantear la necesidad de implementar políticas públicas que las reduzcan y, en particular, un subsidio a la demanda.

El supuesto principal detrás de la propuesta de un subsidio a la demanda es que el precio es el determinante principal en la decisión de tener o no banda ancha, a pesar de que la evidencia para otros países muestra que no siempre es uno de los factores más relevantes (Howick y Whalley, 2008; Lee y Brown, 2008)). Es así como la evaluación de las distintas políticas públicas implementadas en varios países ha llevado a concluir que, en general, aumentar la penetración de banda ancha y uso de Internet requiere un conjunto de políticas que afecten tanto la oferta como la demanda (Cava-Ferreruela y Alabau-Muñoz, 2006) y cuya mezcla responda a la situación específica de cada país o región (Gillet et al., 2006). Este último aspecto es importante, ya que hay gran heterogeneidad entre países respecto a las razones para no tener banda ancha o no usar Internet. Por ejemplo, no tener computador es la causa principal en Portugal, mientras que el precio del servicio lo es en Inglaterra (Choudrie y Dwivedi, 2006). La evidencia de países de la OECD es que el motor principal de suscripción a servicios de banda ancha, por el lado de la demanda, es la predisposición inicial de las personas para usar y adoptar nuevas tecnologías (Cava-Ferreruela y Alabau-Muñoz, 2006), lo cual dificulta el diseño de políticas públicas efectivas para aumentar la penetración de la banda ancha. Por eso es que una política gubernamental intervencionista de magnitud significativa no garantiza resultados y el caso de Singapur es un buen ejemplo de ello (Aizu, 2002).

Un buen diagnóstico es entonces necesario antes de diseñar e implementar políticas públicas que aumenten la penetración de Internet en Chile. En este contexto, este trabajo tiene tres objetivos centrales. El primero es caracterizar la evolución de la "brecha digital" entre los años 
2006 y 2009 en sus dos dimensiones principales: tenencia de computador y conexión en el hogar de banda ancha. El segundo es caracterizar esta brecha en otras dimensiones relevantes, las cuales generalmente no son consideradas por falta de información, como la frecuencia, usos de Internet y lugares de acceso. Finalmente, el tercer objetivo es abrir la discusión respecto a distintas opciones de política pública que, sujetas a una evaluación económica, podrían utilizarse para aumentar la penetración de la banda ancha. Esta discusión está hoy limitada a considerar distintas alternativas de subsidio a la demanda, sin incluso considerar la rentabilidad social de una intervención de ese tipo. A partir de información respecto a las distintas razones para tener o no tener conexión de Internet que los usuarios y no usuarios de Internet revelan, no es claro que esa política sea la mejor alternativa.

El resto del trabajo se organiza de la siguiente manera: en la sección 2 se presentan estadísticas descriptivas de los datos utilizados en el análisis y se discuten los aspectos metodológicos relevantes de la encuesta que los generó. En la sección 3 se caracteriza la evolución de la brecha digital en sus dimensiones de acceso a PC y banda ancha en el hogar, mientras que en la sección 4 se caracteriza la brecha en las dimensiones de uso de Internet (frecuencia y tipos). En la sección 5 se abordan las razones declaradas por los jefes de hogar que pesan en sus decisiones de conexión y uso de Internet y en la sección 6 se presentan las principales conclusiones del trabajo.

\section{Descripción de los datos}

Los datos utilizados en el análisis de este trabajo provienen de una encuesta realizada por el Observatorio Social de la Universidad Alberto Hurtado (OSUAH), la cual tenía como objetivo principal identificar la demanda por Internet en los hogares. En ese sentido ésta es una encuesta única, que entrega información relevante para analizar la brecha digital en Chile y caracterizarla de una forma que la encuesta CASEN no lo permite ${ }^{1}$.

${ }^{1}$ Observatorio Social de la Universidad Alberto Hurtado (OSUAH), "Encuesta sobre Acceso, Uso y Usuarios de Internet Banda Ancha en Chile", 2009, financiada por la Subsecretaría de Telecomunicaciones. 
El universo de la encuesta se define como los jefes de hogar de las poblaciones urbanas de las regiones de Antofagasta, Valparaíso, Biobío y Metropolitana ${ }^{2}$. El diseño muestral es aleatorio, estratificado, de conglomerados y trietápico y para ello se realizó primero un empadronamiento de 3.300 hogares con el objetivo de encuestar finalmente a 1.800. El número final de encuestados fue de 1.717 jefes de hogar, correspondiente a una tasa de respuesta de $95,38 \%$, cuyo detalle por región se presenta en la Tabla $\mathrm{N}^{0} 1^{3}$.

$\begin{array}{ll}\text { TABLA N}^{\circ} 1: & \text { TOTAL DE ENCUESTAS EFECTIVAS, PORCENTAJE DEL TOTAL DE } \\ & \text { ENCUESTAS Y ERROR MUESTRAL POR REGIÓN }\end{array}$

\begin{tabular}{ccc}
\hline Región & $\begin{array}{c}\text { Número de encuestas } \\
\text { efectivas }\end{array}$ & $\begin{array}{c}\text { Porcentaje } \\
\text { del total }\end{array}$
\end{tabular}$\quad$ Error muestral ${ }^{\mathrm{a}}$

\begin{tabular}{lrrr}
\hline II & 414 & $24,1 \%$ & $4,8 \%$ \\
V & 428 & $24,9 \%$ & $4,7 \%$ \\
VIII & 451 & $26,3 \%$ & $4,6 \%$ \\
R.M. & 424 & $24,7 \%$ & $4,8 \%$ \\
Total & 1.717 & $100 \%$ & $2,4 \%$ \\
\end{tabular}

${ }^{a}$ Error muestral para muestra aleatoria al interior de cada estrato, con varianza máxima y nivel de confianza igual a $95 \%$.

Fuente: OSUAH (2009), Tabla $\mathrm{N}^{\mathrm{o}} 1$.

Las Tablas Nos. 2 y 3 a continuación presentan estadísticas demográficas y económicas básicas de los jefes de hogar encuestados.

Para ver la evolución de la brecha digital en sus variables principales (e.g., tenencia de PC y conexión de banda ancha), los datos de la encuesta OSUAH (Observatorio Social de la Universidad Alberto Hurtado) se comparan en la siguiente sección con los de la CASEN del año $2006^{4}$.

${ }^{2}$ Se define jefe de hogar como quien declara en la encuesta ser el principal aportante del ingreso del hogar. (2008).

${ }^{3}$ Para mayores detalles técnicos de la encuesta ver OSUAH (2009) y OSUAH

${ }^{4}$ Para información sobre las características centrales de la encuesta CASEN puede visitarse el sitio del Ministerio de Planificación, www.mideplan.cl. 
TABLA N ${ }^{\circ}$ 2: $\quad$ PORCENTAJE DE JEFES DE HOGAR/HOGARES SEGÚN VARIABLES DE SEGMENTACIÓN

Variable Porcentaje

Sexo del jefe de hogar

Hombre $\quad 48,8$

Mujer $\quad 51,2$

Edad del jefe de hogar

Entre 15 y 29 años

Entre 30 y 44 años

Entre 45 y 59 años

De 60 años y más

Educación del jefe de hogar

Básica incompleta

Básica completa

13,1

Media incompleta

19,2

Media completa

29,7

Superior incompleta

Superior completa

Situación ocupacional

Trabajador (asalariado o independiente) $\quad 56,9$

Busca trabajo 3,1

Jubilado/a $\quad 15,8$

$\begin{array}{ll}\text { Labores del hogar } & 21,7\end{array}$

Estudiante $\quad 1,7$

Otra 0,9

Presencia de hijos en edad escolar

Sin presencia de hijos entre 6 y 18 años $\quad 57,7$

Con presencia de hijos entre 6 y 18 años $\quad 42,3$

Fuente: OSUAH (2009), Tabla № 2.

TABLA N ${ }^{\circ}$ 3: $\quad$ MEDIA DE INGRESO AUTÓNOMO E INGRESO PER CÁPITA DE LOS HOGARES

\begin{tabular}{lccccc}
\hline Característica del hogar & $\mathrm{N}^{\circ}$ & Media & $\begin{array}{c}\text { Desviación } \\
\text { estándar }\end{array}$ & Mín. & Máx. \\
& & & & \\
\hline Ingreso autónomo del hogar & 1.265 & 394.325 & $471.872,8$ & 8.000 & 6.000 .000 \\
Ingreso per cápita & 1.265 & 120.287 & $150.140,9$ & 2.000 & 2.000 .000 \\
\hline
\end{tabular}

Fuente: OSUAH (2009), Tabla No 3. 


\section{Las brechas de acceso a computador y banda ancha}

La denominada "brecha digital" (digital divide en inglés) se entiende como las diferencias en la capacidad de acceso a las tecnologías de información y comunicaciones de distintos grupos de población en un mismo país o a nivel internacional. Una de las dimensiones de esta brecha, probablemente la más estudiada, dice relación con el acceso a computadores y con el acceso efectivo a conexiones de banda ancha. En esta sección exploramos estas brechas en distintas dimensiones y cómo han evolucionado en los últimos años.

\section{La brecha de acceso al PC y banda ancha}

A partir de los datos de la encuesta realizada y de los datos de la encuesta CASEN de 2006 es posible concluir que ha habido importantes progresos en cuanto a aumento de la penetración de computadores en los hogares. En las regiones estudiadas la penetración total ha aumentado de alrededor del $37 \%$ al $61 \%$ en poco más de dos años ${ }^{5}$.

Este progreso ha sido importante en todos los quintiles de ingresos, aunque los aumentos más importantes en términos absolutos se verifican en los tres quintiles intermedios, tal como lo revela el Gráfico $\mathrm{N}^{\mathrm{o}} 1$. En éste la altura total de las barras indica el porcentaje de hogares con PC por quintil para los años 2006 y 2009. Los segmentos alrededor de la parte superior de la barra indican intervalos de confianza del $95 \%$ para el total de individuos con PC (nótese que éstos son menores en el dato del año 2006 por provenir de una encuesta - CASEN — con mayor representatividad $)^{6}$.

Un patrón similar de crecimiento ha experimentado la penetración de la banda ancha, que subió de un $18 \%$ a casi el $41 \%$ en el mismo período, aunque en este caso los aumentos más importantes ocurrieron en los tres quintiles de ingreso superior, tal como ilustra la altura de las barras grises en el Gráfico $\mathrm{N}^{\circ} 1$. En este caso los segmentos más oscu-

${ }^{5}$ El relevamiento de la encuesta CASEN 2006 corresponde al mes de noviembre, en tanto que para la Encuesta sobre Acceso, Uso y Usuarios de Internet Banda Ancha en Chile el levantamiento de datos se realizó entre los meses de diciembre de 2008 y marzo de 2009.

${ }^{6}$ En todos los gráficos los "segmentos" alrededor del valor indicado por cada barra marcan el intervalo de confianza del $95 \%$. Tanto las medias muestrales como los intervalos de confianza han sido calculados con estimadores de Jacknife considerando la estructura del diseño muestral de la encuesta. 
GRÁFICO No $1: \quad$ HOGARES CON PC Y CON BANDA ANCHA POR QUINTIL DE INGRESO (2006 Y 2009) (\%)

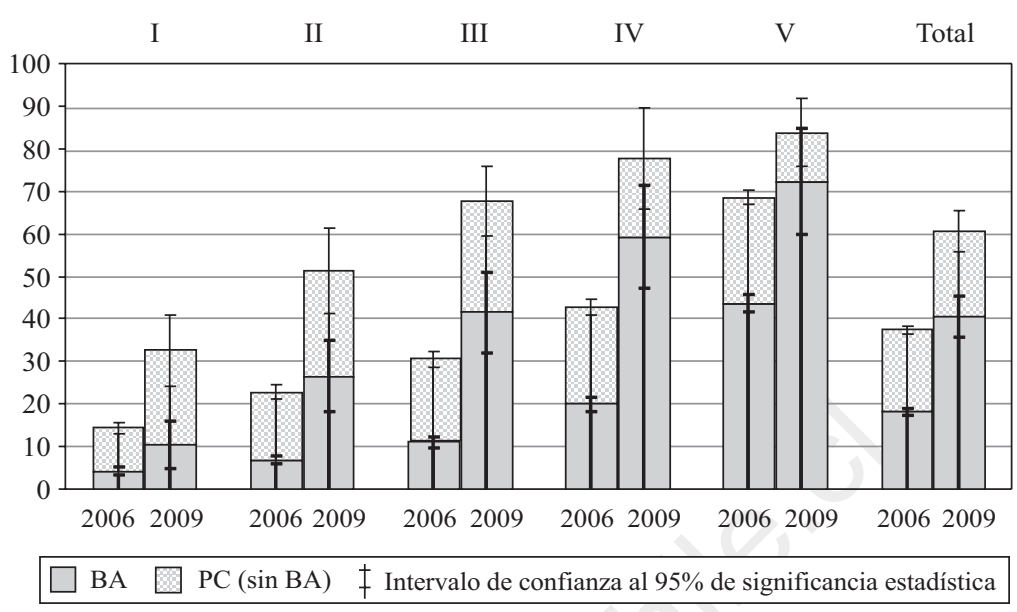

Fuentes: Elaboración propia en base a Encuesta sobre Acceso, Uso y Usuarios de Internet Banda Ancha en Chile 2009 y encuesta CASEN 2006.

ros alrededor de la altura total de la barra gris representan, nuevamente, intervalos de confianza del $95 \%$.

Aunque visualmente no es tan evidente en el Gráfico $\mathrm{N}^{\circ} 1$, los valores muestran también un aumento en la penetración de la banda ancha entre quienes tienen PC. Es decir, entre el año 2006 y 2009 los suscriptores de banda ancha medidos como porcentaje del total de hogares con PC aumentaron, particularmente en los tres quintiles intermedios.

Tanto en la penetración de la banda ancha como en la de computadores se aprecia que la brecha entre el quintil más rico y el más pobre, medida en valores absolutos, se ha ampliado, aunque si medimos esta misma brecha en términos relativos claramente se ha reducido (de 5 veces a 2,5 en el caso de los computadores y de 10 veces a 6,6 en el caso de la banda ancha). Como sea y más allá de las diferencias por ingreso que persisten, es destacable el crecimiento que ha habido en los últimos años en todos los grupos.

Más allá de las marcadas diferencias que subsisten, desde una perspectiva de políticas públicas cabe preguntarse si las diferencias observadas en el "consumo" de este servicio (acceso a banda ancha en el hogar) son muy distintas de las observadas en el consumo de otros bie- 
nes o servicios (e.g., automóviles) y por qué el caso de la banda ancha ameritaría la intervención del Estado. A modo de ejemplo, de acuerdo a la encuesta CASEN 2006, el porcentaje de hogares con automóvil en el primer quintil era de $11,4 \%$, en tanto que para el quintil de ingresos superior era del $65 \%$. Si bien evaluar la magnitud de distintas brechas existentes en Chile y la rentabilidad social de una potencial política pública para reducirlas va más allá de los objetivos de este trabajo, en la sección de conclusiones se plantean algunas reflexiones adicionales respecto a la evaluación de brechas en el caso de la banda ancha.

El Gráfico $\mathrm{N}^{\mathrm{o}} 2$, al igual que el anterior, muestra la evolución de la penetración de los PC y de la banda ancha desde una perspectiva regional. En este gráfico destacan de alguna manera la performance más pobre de la Octava Región, tanto en lo que respecta a penetración del PC como de la banda ancha, y el crecimiento importante observado para ambas variables en la Quinta Región.

¿Cuáles factores, más allá del ingreso del hogar, están relacionados con las decisiones de tener PC y banda ancha en el hogar? Los siguientes gráficos ilustran la relevancia de dos factores adicionales al ingreso: la educación del jefe de hogar y la presencia en el hogar de escolares o jóvenes en edad universitaria.

GRÁFICO No ${ }^{\circ}$ : HOGARES CON PC Y CON BANDA ANCHA POR REGIÓN (2006 Y 2009) (\%)

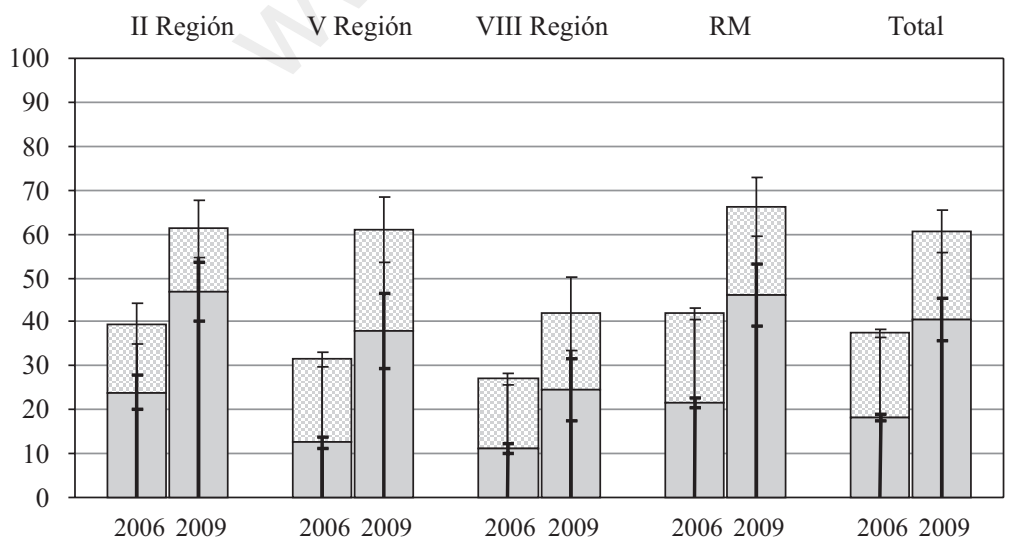

BA $\square$ PC (sin BA) $\ddagger$ Intervalo de confianza al 95\% de significancia estadística

Fuentes: Elaboración propia en base a Encuesta sobre Acceso, Uso y Usuarios de Internet Banda Ancha en Chile 2009 y encuesta CASEN 2006. 
El Gráfico No 3 indica que para el año 2009 el 92\% de los hogares cuyos jefes cuentan con educación superior completa tiene PC, lo que contrasta fuertemente con los porcentajes de 36 y $60 \%$ cuando los jefes tienen educación básica incompleta y completa respectivamente. Si bien la brecha absoluta de acceso a PC entre el grupo con más educación formal y los otros se ha mantenido relativamente constante o se ha reducido levemente, en el contexto de una mayor penetración en todos los grupos esto significa una reducción importante en términos relativos.

Respecto a las brechas en términos de acceso a banda ancha, éstas -al igual que ocurre cuando comparamos por quintiles - se han ampliado en términos absolutos y reducido en términos relativos en los últimos tres años.

Es importante destacar (aunque no es evidente a partir del Gráfico $\mathrm{N}^{\circ}$ 3) que la educación es un factor relevante para el acceso a PC cuyo efecto va más allá del asociado al ingreso. Es decir, en un mismo nivel de ingreso, los hogares cuyos jefes alcanzaron mayores niveles de educación tienen una probabilidad mayor de contar con PC.

Por último, el Gráfico $N^{o} 4$ ilustra la diferencia de penetración tanto de PC como de banda ancha entre tres grupos: hogares sin hijos,

GRÁFICO No 3: HOGARES CON PC Y CON BANDA ANCHA POR NIVEL EDUCATIVO (2006 Y 2009) (\%)

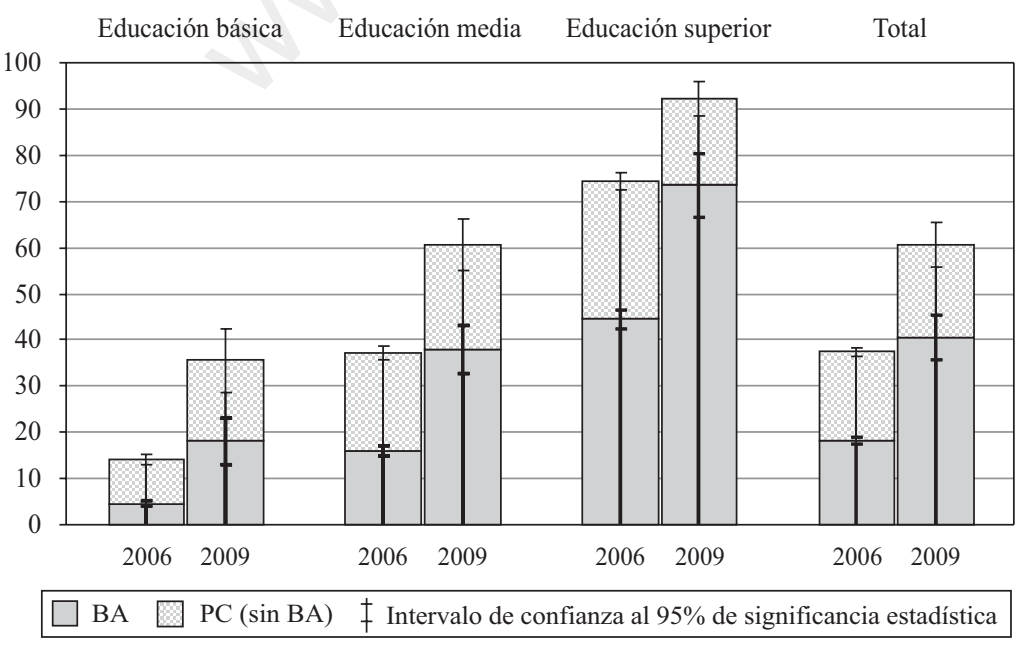

Fuentes: Elaboración propia en base a Encuesta sobre Acceso, Uso y Usuarios de Internet Banda Ancha en Chile 2009 y encuesta CASEN 2006. 
GRÁFICO No 4: HOGARES CON PC Y CON BANDA ANCHA, SEGÚN EDAD DE HIJOS Y JEFE DE HOGAR (2009) (\%)

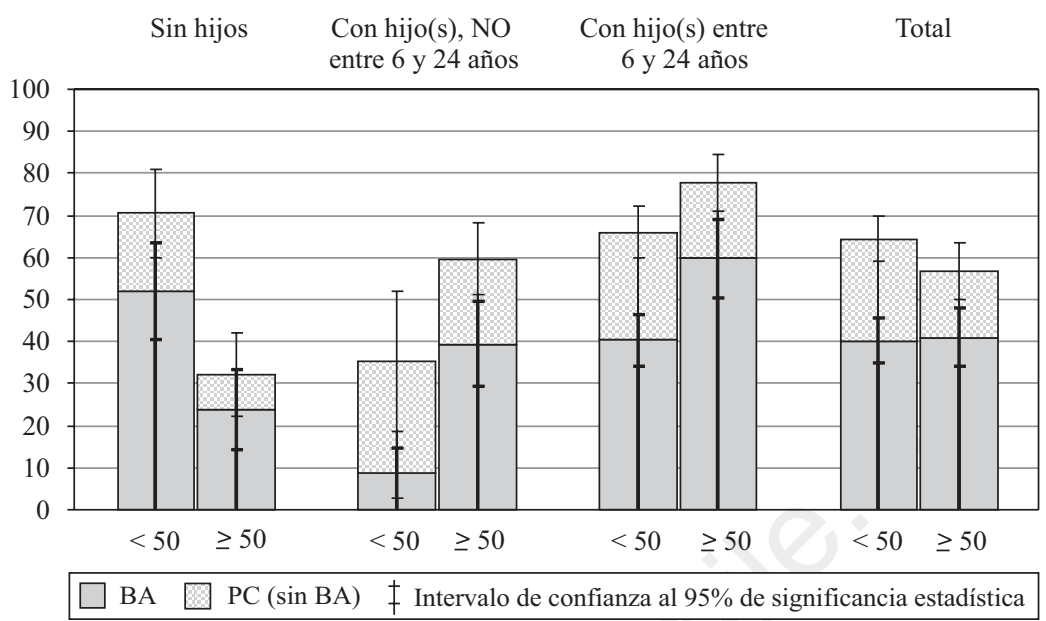

Fuente: Elaboración propia en base a Encuesta sobre Acceso, Uso y Usuarios de Internet Banda Ancha en Chile 2009.

hogares con al menos un hijo en edad escolar o universitaria (entre 6 y 24 años) y hogares con hijos pero no en este rango de edad, distinguiendo si el jefe de hogar es mayor o menor de 50 años. Los datos corresponden solamente al año 2009.

En aquellos hogares con jefes de más de 50 años, la presencia de hijos en el hogar ( $\mathrm{y}$ en particular en edad de estudiar) está fuertemente asociada a una mayor tasa de penetración del PC y, aun más marcada, de la banda ancha.

En los hogares cuyos jefes son menores de 50 años se observa un efecto similar al comparar hogares con hijos entre 6 y 24 años y hogares con hijos pero no en este rango de edad. Cuando se trata de hogares sin hijos, el que los jefes sean más jóvenes es también un elemento fuertemente asociado a mayor penetración del PC y la banda ancha.

\section{Las brechas de uso}

Un análisis que resulta interesante (y no es tan frecuente como el de acceso) es el de las brechas de uso de Internet. En términos de la discusión realizada en la introducción del trabajo y el rol de las externali- 
dades como posible justificación económica al fomento de la banda ancha, es evidente que las mencionadas externalidades están relacionadas casi exclusivamente con el uso del servicio y no con la mera conexión.

Si bien la brecha de uso está relacionada en parte con la brecha de acceso, puede diferir de ésta por las diversas opciones de conexión a Internet fuera del hogar (e.g., trabajo, centros de Internet, etc.) y por las decisiones de uso al interior del hogar (ya que no necesariamente todos los miembros de un hogar con conexión hacen uso del servicio) ${ }^{7}$.

Esta dimensión del análisis es complementaria a la del acceso y muy importante para el diseño de políticas. De poca utilidad sería una política que facilite el acceso a la banda ancha si éste no se tradujera en uso - ojalá relativamente productivo- de Internet. De igual manera, una política que facilite el acceso en el hogar podría tener un menor impacto si los miembros de éste tienen sustitutos razonables como acceso en el lugar de estudio o trabajo, o en bibliotecas y centros comunitarios.

La información presentada en los siguientes gráficos permite analizar las diferencias por quintil en el uso de Internet en cuatro dimensiones diferentes: si lo utiliza o no, cuán frecuentemente lo utiliza, dónde lo utiliza y qué tipo de aplicaciones son las más frecuentes. Resulta interesante notar que algunas de las diferencias observadas entre los quintiles persisten incluso cuando se limita el análisis de los patrones de uso a aquellos hogares que tienen conexión de banda ancha. Es decir, existen diferencias importantes incluso entre quienes suscriben el servicio de banda ancha en el hogar.

La porción gris de las diferentes barras del Gráfico $\mathrm{N}^{0} 5$ ilustra las diferencias existentes por quintiles en el porcentaje de jefes de hogar que declaran utilizar Internet, dentro o fuera de su hogar. Entre los extremos de la distribución de ingreso, la brecha es de más de 50 puntos porcentuales: casi $74 \%$ para el quintil más rico y apenas $17 \%$ para el más pobre en el año 2009. La evolución de esta brecha muestra claramente que los aumentos absolutos en las tasas de uso de Internet más relevantes se han producido en los tres quintiles intermedios (particularmente en los quintiles III y IV), que han reducido su brecha respecto al quintil más rico en términos absolutos. La brecha absoluta del quintil

${ }^{7}$ Di Maggio et al. (2004) muestran la existencia de diferencias importantes en la cantidad y tipo de uso entre usuarios de Internet. De igual forma, Ono y Zavodny (2003) muestran diferencias significativas entre tipos de usuarios en cinco países (Suecia, Estados Unidos, Japón, Corea del Sur y Singapur). 
GRÁFICO N ${ }^{\circ}$ 5: TASA DE USO DE INTERNET GENERAL Y DE QUIENES TIENEN CONEXIÓN DE BANDA ANCHA.

JEFES DE HOGAR POR QUINTIL DE INGRESO (2006 Y 2009) (\%)

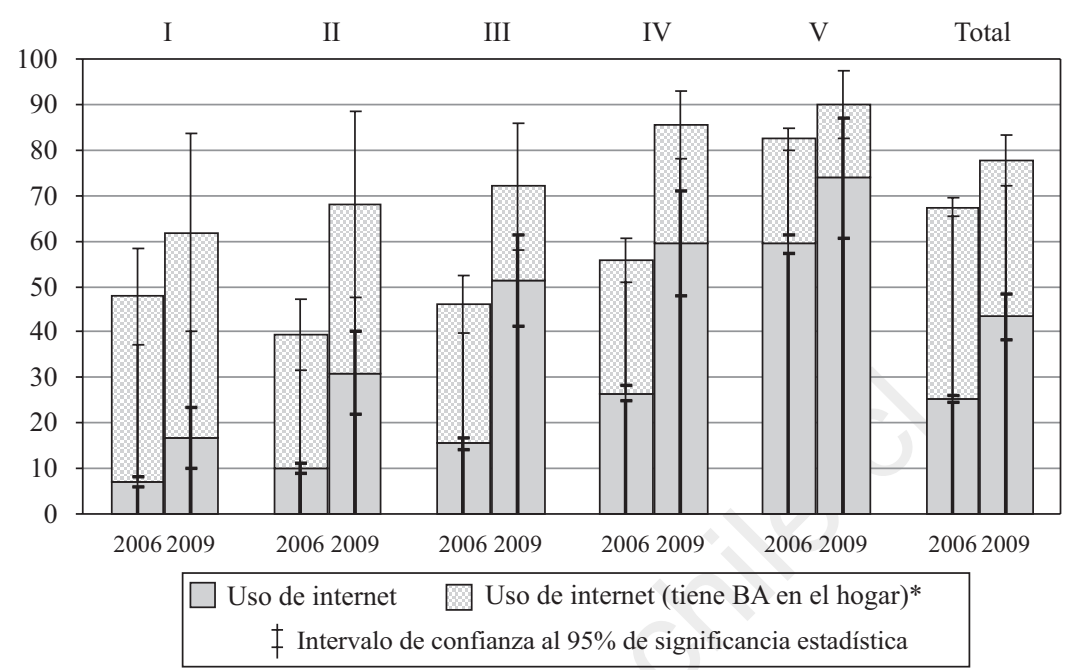

(*) El porcentaje de jefes de hogar usuarios de internet entre quienes tienen conexión de banda ancha corresponde, para cada quintil y año, a la altura total de la barra respectiva.

Fuente: Elaboración propia en base a Encuesta sobre Acceso, Uso y Usuarios de Internet Banda Ancha en Chile 2009.

más pobre respecto a cualquier otro, en cambio, se ha ampliado considerablemente.

Resulta interesante que la brecha en el uso de Internet observada para jefes de hogar de los diversos quintiles no desaparece, aunque obviamente sí se reduce, cuando consideramos solamente los jefes de aquellos hogares que sí tienen conexión de banda ancha. En este mismo Gráfico $\mathrm{N}^{\circ} 5$, la altura total de las barras representa la tasa de uso de los jefes de aquellos hogares que sí tienen conexión de banda ancha. Es decir, más allá de las diferencias importantes en la tenencia de conexión de banda ancha por quintil, aun entre quienes tienen conexión existe una diferencia en la tasa de uso de los jefes de hogar. Las diferencias observadas en el Gráfico $\mathrm{N}^{\circ} 5$ para el año 2009 son importantes y significativas al 5\% cuando se compara cualquiera de los tres quintiles de menores ingresos con cualquiera de los dos superiores. Estas diferencias observadas entre los quintiles ratifican un punto que es fundamen- 
tal tener en cuenta a la hora de diseño de políticas: el acceso a la banda ancha no necesariamente se traduce en uso de la misma ${ }^{8}$.

Nótese que la tasa de uso, cuando se tiene conexión de banda ancha, ha aumentado en todos los quintiles. Ello no tendría por qué ser necesariamente así ya que, en principio, hay dos efectos que actúan en direcciones opuestas: por un lado, en el tiempo transcurrido entre ambas encuestas (algo más de dos años) seguramente más miembros del hogar han aprendido a utilizar el servicio y ello explicaría un aumento. Por otro, sin embargo, es razonable pensar que los hogares que se han conectado en los últimos años son hogares con un menor interés que quienes ya lo estaban y, por ende, con una menor tasa de utilización, lo que podría haber explicado una baja en la tasa de uso condicional. El primer efecto parece dominar en todos los quintiles.

El Gráfico $\mathrm{N}^{\mathrm{o}} 6$ ilustra un punto adicional: incluso entre quienes son usuarios de banda ancha y tienen conexión en el hogar podrían existir diferencias por quintil en las frecuencias de uso. Como ya se mencionó, este tipo de datos resulta relevante por cuanto las potenciales externalidades de la banda ancha están asociadas al uso de la misma y, en gran medida también, a la frecuencia de su uso9.

Por ejemplo, el porcentaje de jefes de hogar que teniendo conexión de banda ancha la utilizan al menos cinco veces a la semana es cercano al $34 \%$ en el quintil más pobre, mientras que en los dos quintiles más ricos dicho porcentaje es cercano al 70\%. Para otras frecuencias de uso las diferencias observadas son más pequeñas y, dado el tamaño muestral (y los consiguientes errores estándar), en muchos casos no significativas.

En cuanto a los lugares de conexión, el Gráfico $\mathrm{N}^{0} 7$ ilustra las diferencias existentes entre los diversos quintiles. Éstas resultan esperables: el uso de Internet en el hogar y en el trabajo es significativamente más frecuente en los quintiles de ingresos más altos, en tanto que el uso en centros pagados de Internet es más frecuente en los quintiles de menor ingreso. Respecto a las demás opciones, como establecimientos educacionales, infocentros gratuitos o lugares con red inalámbrica gra-

${ }^{8} \mathrm{Si}$ se considera que existe un efecto de autoselección entre quienes deciden conectarse, entonces el porcentaje de jefes de hogar por quintil que efectivamente utilizaría Internet en caso de que, por ejemplo, se le regalase la suscripción al servicio, sería menor que el identificado en el Gráfico $\mathrm{N}^{\mathrm{o}} 5$.

${ }^{9}$ Naturalmente la relevancia de la frecuencia depende de la aplicación de que se trate. Por ejemplo, para el pago de imposiciones, una frecuencia mayor que un mes no tiene mucho sentido. 
GRÁFICO No 6: FRECUENCIA ACUMULADA DE USO DE INTERNET EN EL ÚLTIMO MES. JEFES DE HOGAR USUARIOS DE INTERNET CON CONEXIÓN DE BA EN EL HOGAR (2009) (\%)

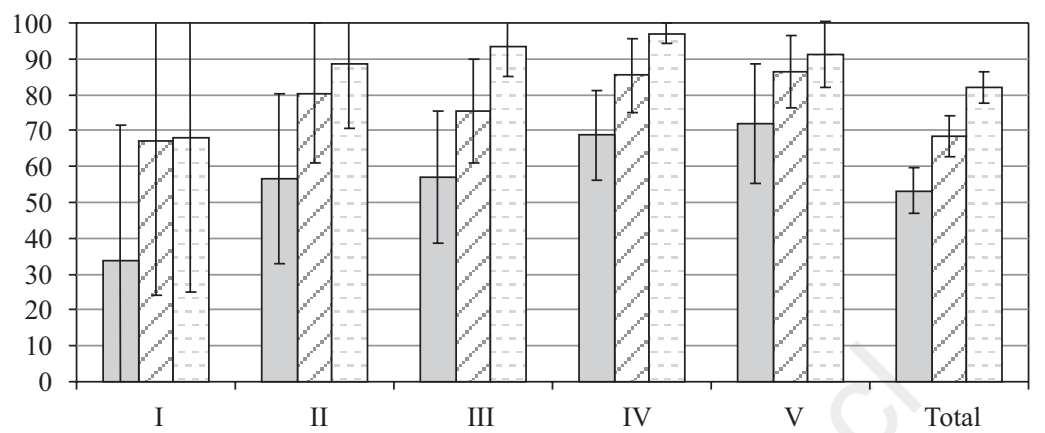

Al menos 5 días a la semana $\quad \square \mathrm{Al}$ menos 3 días a la semana $\quad \square \mathrm{Al}$ menos una vez a la semana † Intervalo de confianza al $95 \%$ de significancia estadística

Fuente: Elaboración propia en base a Encuesta sobre Acceso, Uso y Usuarios de Internet Banda Ancha en Chile 2009.

GRÁFICO No 7 : LUGARES DE CONEXIÓN DE JEFES DE HOGAR USUARIOS, POR QUINTIL DE INGRESO (AÑO 2009) (\%)
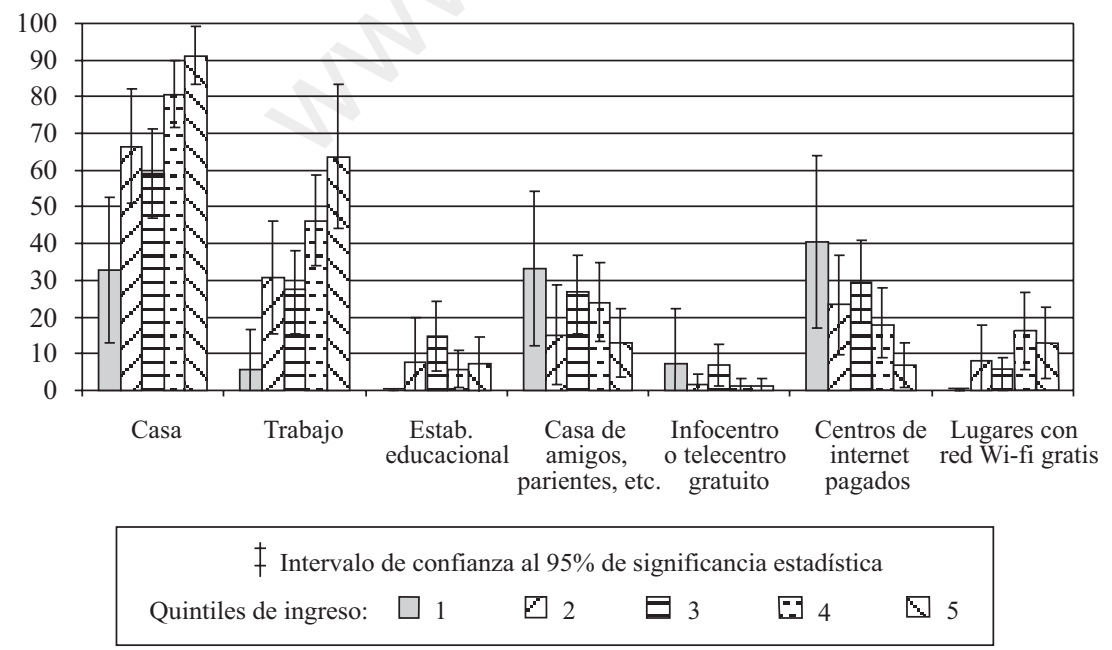

Fuente: Elaboración propia en base a Encuesta sobre Acceso, Uso y Usuarios de Internet Banda Ancha en Chile 2009. 
tuita, no es posible concluir que existan diferencias significativas entre los distintos quintiles de ingreso (esto, lógicamente, no significa que no haya diferencias, pero a partir de la muestra y los errores de la misma no es posible afirmarlo).

Más allá de las posibles diferencias entre grupos, del Gráfico $\mathrm{N}^{\mathrm{o}} 7$ se desprende también que el impacto en términos cuantitativos de centros de conexión gratuitos es relativamente bajo comparado con, por ejemplo, los infocentros pagados. Esto es cierto especialmente para los quintiles inferiores.

Finalmente, los siguientes gráficos ilustran una dimensión adicional de la brecha digital que dice relación con el uso más o menos "productivo" de Internet que pudieran hacer los distintos tipos de usuarios.

El Gráfico $\mathrm{N}^{\circ} 8 \mathrm{~A}$ muestra para seis actividades relacionadas fundamentalmente con el ocio los diferentes niveles de utilización por quintil. Como puede apreciarse, para estas actividades recreativas las tasas de utilización no difieren mayormente entre quintiles. Las diferencias, por quintiles, en las tasas promedio de uso no resultan significati-

GRÁFICO No 8A: USOS DE LA BANDA ANCHA POR QUINTIL DE INGRESO. JEFES DE HOGAR USUARIOS (2009) (\%)
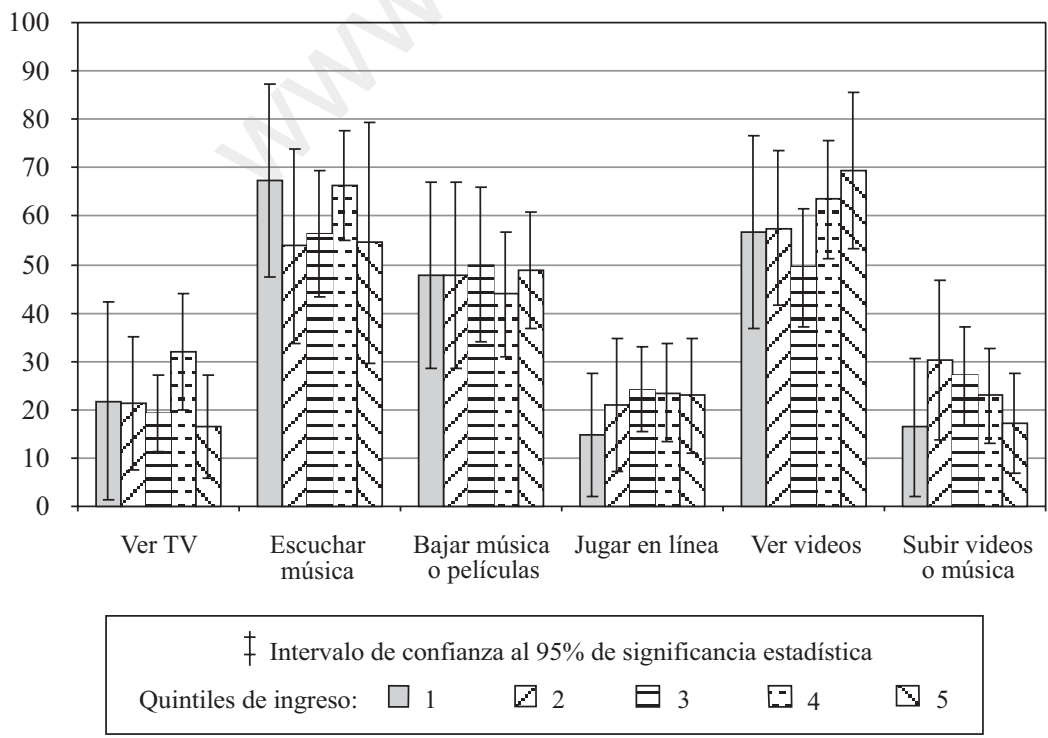

Fuente: Elaboración propia en base a Encuesta sobre Acceso, Uso y Usuarios de Internet Banda Ancha en Chile 2009. 
GRÁFICO No 8B: USOS DE LA BANDA ANCHA POR QUINTIL DE INGRESO. JEFES DE HOGAR USUARIOS (2009) (\%)

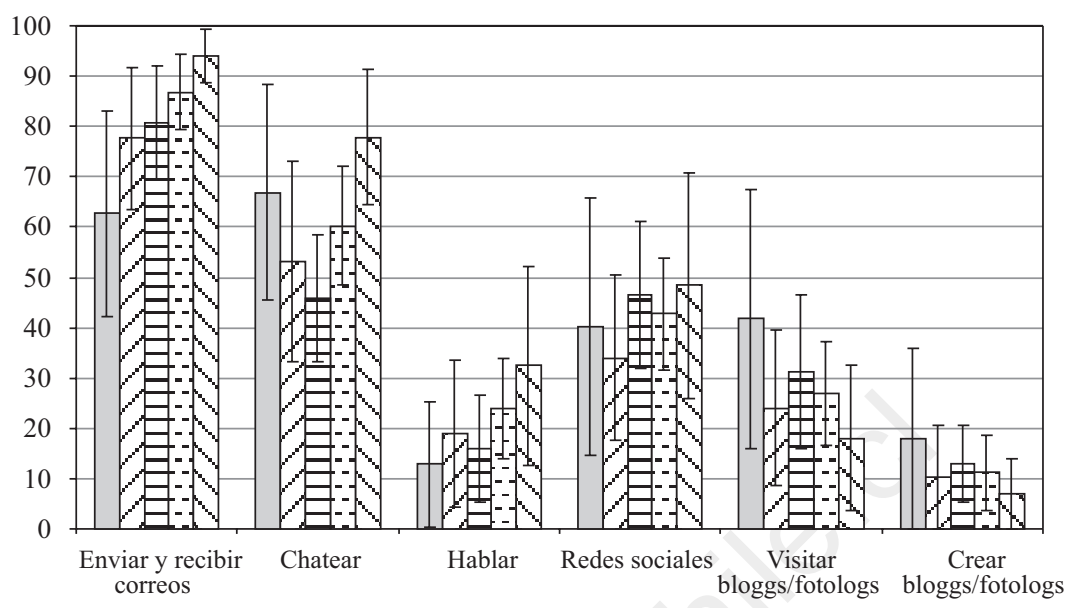

\begin{tabular}{|c|c|c|c|c|c|}
\hline \multicolumn{6}{|c|}{ † Intervalo de confianza al 95\% de significancia estadística } \\
\hline Quintiles de ingreso: & $\square 1$ & Q 2 & $\boxminus 3$ & $E 4$ & 5 \\
\hline
\end{tabular}

Fuente: Elaboración propia en base a Encuesta sobre Acceso, Uso y Usuarios de Internet Banda Ancha en Chile 2009.

vas estadísticamente (debe recalcarse que tanto en este gráfico como en los subsiguientes se considera, para cada quintil, solamente al subgrupo de jefes de hogar que son usuarios de Internet).

Como lo ilustra el Gráfico $\mathrm{N}^{\circ} 8 \mathrm{~B}$, para actividades relacionadas más con las comunicaciones entre personas, sea directamente o a través de redes sociales, las diferencias en tasas de uso por quintil no son importantes, a excepción de la utilización del correo electrónico, que es más frecuente en los quintiles de mayor ingreso y, en menor medida, el "hablar" por Internet (aunque en este último caso, dada la magnitud de los errores estándar, las diferencias entre algunos quintiles no son estadísticamente significativas).

Finalmente, el Gráfico $\mathrm{N}^{\circ} 8 \mathrm{C}$ ilustra los patrones de usos para actividades "más productivas", como podrían ser las que involucran transacciones económicas y de búsqueda de información. Como es esperable, en las que involucran transacciones económicas la brecha es muy marcada, lo que puede obedecer más a un diferente grado de bancarización entre los diferentes quintiles que a una diferente habilidad y/o interés en la realización de estas actividades. 
GRÁFICO No 8C: USOS DE LA BANDA ANCHA POR QUINTIL DE INGRESO. JEFES DE HOGAR USUARIOS (2009) (\%)

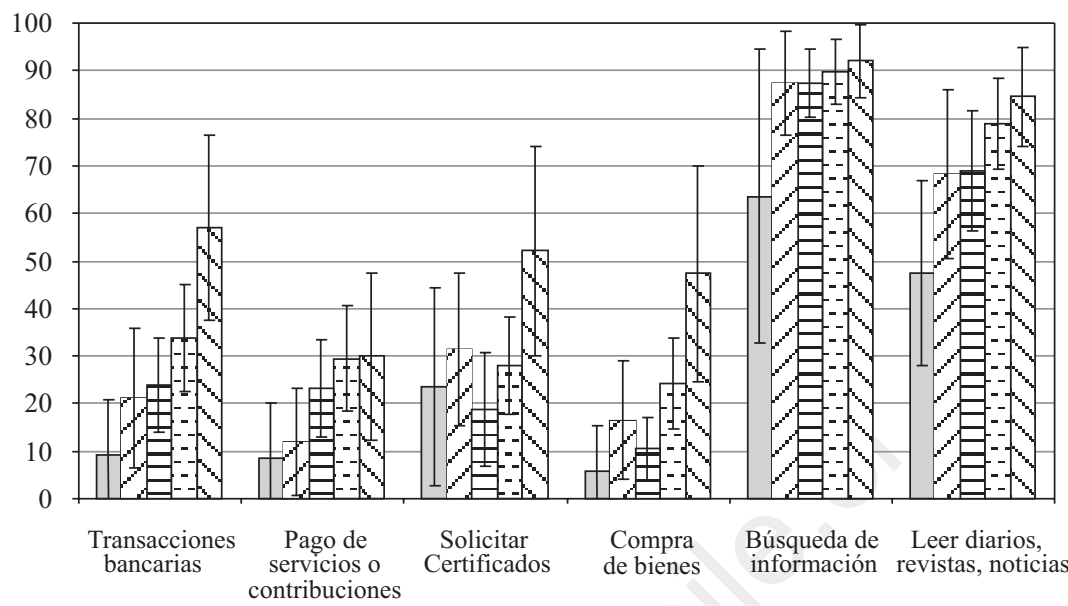

$f$ Intervalo de confianza al $95 \%$ de significancia estadística
Quintiles de ingreso: $\square 1 \quad \square l_{2} \quad \square_{3} \quad \square_{4} \quad \square 5$

Fuente: Elaboración propia en base a Encuesta sobre Acceso, Uso y Usuarios de Internet Banda Ancha en Chile 2009.

En lo que hace a la utilización de Internet para búsqueda de información y para la lectura de diarios y noticias, también existe una brecha de uso importante, en particular entre el primer quintil y los demás.

A manera de síntesis, es posible afirmar entonces que la medición de la brecha digital por quintiles de ingreso se vería claramente subestimada si se utiliza como indicador la penetración de la banda ancha en hogares. En esta sección se muestra evidencia clara de que, aun entre quienes tienen conexión de banda ancha, existen importantes diferencias por quintil de ingreso en cuanto a que el jefe de hogar haga o no uso de la misma, en cuanto a la frecuencia con que hace uso de la banda ancha y, en menor medida, en cuanto a los tipos de aplicaciones utilizados.

\section{Las razones detrás de las decisiones de conexión y uso de Internet}

En su conjunto, el análisis y los gráficos de las dos secciones anteriores ilustran al menos tres puntos que deben considerarse a la hora 
del diseño de políticas públicas: 1) ha habido en los últimos dos años un crecimiento importante en la penetración de la banda ancha que es transversal a niveles de ingreso, educación y también geográfico; 2) este importante crecimiento ha ampliado las brechas absolutas entre quintiles, pero éstas han disminuido en términos relativos, y 3) la "brecha digital" tiene más facetas que sólo el acceso o conexión de banda ancha en el hogar: existen importantes diferencias en tasas de uso y frecuencias de uso y, en menor medida, en tipos de uso. Desde la perspectiva de política pública (no ciertamente de la empresa privada) es más relevante la brecha de uso de Internet con todas sus dimensiones (el ser usuario o no, la frecuencia de uso y los tipos de usos más frecuentes o más valorados) que la simple brecha de acceso.

Para el diseño de cualquier política pública que busque promover el uso de la banda ancha en Chile resulta clave entender los factores que afectan las decisiones tanto de quienes son usuarios de banda ancha como de quienes no lo son. La encuesta utilizada permite abordar de manera directa los factores asociados a las decisiones de suscripción a servicios de banda ancha y uso de Internet. En esta sección se sintetizan los principales elementos detectados, discriminando en algunas de las respuestas según el nivel de ingreso de los hogares.

La Tabla $\mathrm{N}^{\circ} 4$ resume las respuestas de los jefes de aquellos hogares que no tienen conexión de banda ancha respecto a la principal razón y la segunda razón más importante por las cuales no tienen conexión. La no tenencia de PC es el factor de mayor preponderancia, siendo la principal razón para el $42 \%$, y la segunda más importante para

TABLA N ${ }^{\circ}$ 4: $\quad$ PRINCIPALES RAZONES POR LAS QUE NO TIENE CONEXIÓN DE BANDA ANCHA EN EL HOGAR (2009) (\%)

\begin{tabular}{|c|c|c|c|c|c|}
\hline \multirow[t]{3}{*}{ Principal razón } & \multicolumn{4}{|c|}{ Segunda razón } & \multirow[b]{2}{*}{ Total } \\
\hline & No tiene PC & $\begin{array}{c}\text { Es muy } \\
\text { caro }\end{array}$ & $\begin{array}{r}\text { Desinterés } \\
\text { desconocimi }\end{array}$ & Otra & \\
\hline & \multicolumn{4}{|c|}{ En porcentaje } & \\
\hline No tiene PC & 0 & 29 & 8 & 5 & 42 \\
\hline Es muy caro & 5 & 0 & 10 & 17 & 31 \\
\hline Desinterés o desconocimiento & 2 & 3 & 8 & 6 & 19 \\
\hline Otra & 1 & 2 & 2 & 4 & 8 \\
\hline Total & 7 & 34 & 27 & 32 & 100 \\
\hline
\end{tabular}

Fuente: Elaboración propia en base a Encuesta sobre Acceso, Uso y Usuarios de Internet Banda Ancha en Chile 2009. 
un 7\% adicional. En cualquier caso, la suma de estos porcentajes es inferior al $66 \%$, que es el porcentaje que, del total que no tienen conexión, no tienen PC.

En segundo orden de importancia está el costo del servicio de banda ancha. Para el 31\% de quienes no tienen conexión es la principal razón y para el 34\% la segunda. Si se considera adicionalmente que para un porcentaje muy importante, alrededor del $60 \%$, de quienes no tienen PC la principal causa es su costo, entonces claramente el factor monetario es la razón de fondo principal por la cual no tienen conexión.

También es importante destacar la relevancia de un tercer factor, que tiene que ver con el desinterés o desconocimiento respecto al servicio de banda ancha ${ }^{10}$. Para casi el $40 \%$ de quienes no tienen banda ancha la razón principal o la segunda más importante es el desconocimiento o desinterés por el servicio.

Este último dato es altamente relevante para el diseño de cualquier política pública que busque promover el desarrollo de la banda ancha. Que el factor monetario sea de primer orden de importancia no debe sorprender: seguramente si se preguntase, por ejemplo, la principal razón para no tener un automóvil entre quienes no tienen uno el factor monetario (sea por su costo inicial o de mantenimiento) sería fundamental si no excluyente. Sin embargo, que el $40 \%$ declare no estar muy interesado o desconocer cómo utilizar el servicio es un indicio claro de que una política de subsidio al PC o a la conexión de banda ancha podría resultar insuficiente y, muy probablemente, ineficiente por cuanto una fracción importante de quienes podrían recibir el subsidio harían un uso marginal del servicio. Ello, lógicamente, invalidaría una de las principales razones para la intervención de política, cual es la existencia de externalidades, asociadas fundamentalmente al uso de la banda ancha y no a la mera conexión.

Contrariamente a lo que podría especularse a priori, las razones por las que no se tiene una conexión de banda ancha no difieren demasiado por quintil de ingreso, por lo que las consideraciones anteriores son válidas incluso si las políticas de subsidio fueran focalizadas. En particular, tal como ilustra el Gráfico $\mathrm{N}^{\circ} 9$, los porcentajes de respuestas

${ }^{10}$ En esta categoría de "Desinterés o desconocimiento" se incorporaron varios tipos de respuestas a la pregunta "¿Cuál es la principal (segunda, tercera) razón por la cual no tiene Internet en el hogar?": concretamente, "No lo necesito por ahora", "No sé cómo usarla", "No sé para qué sirve" y "No me interesa por ahora". 
GRÁFICO N ${ }^{\circ}$ 9: PRINCIPAL RAZÓN PARA NO TENER CONEXIÓN DE BA POR QUINTIL DE INGRESO (2009) (\%)

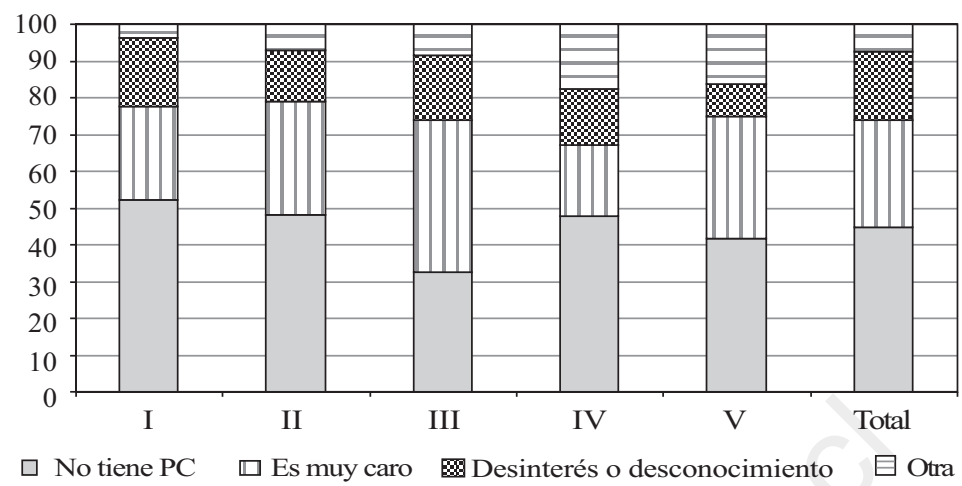

Fuente: Elaboración propia en base a Encuesta sobre Acceso, Uso y Usuarios de Internet Banda Ancha en Chile 2009.

asociadas a desinterés o desconocimiento no son tan diferentes entre los diferentes grupos.

A todos los jefes de hogar, tanto usuarios como no usuarios de Internet, se les consultó también si les gustaría utilizar o utilizar más frecuentemente Internet, por qué no lo hacen y qué los animaría a hacerlo.

En en el Gráfico $N^{0} 10$ se presenta la distribución de respuestas a la pregunta "¿Le gustaría usar o usar más Internet de lo que lo hace actualmente?", distinguiendo según si el jefe de hogar es o no usuario de Internet y si tiene o no conexión de banda ancha en el hogar.

Como se observa en el Gráfico $\mathrm{N}^{\circ} 10$, los dos grupos más grandes corresponden a los que no tienen banda ancha y no son usuarios de Internet $(47,6 \%)$ y los que tienen banda ancha y son usuarios $(31,6 \%)$. Sin embargo, tal como se esperaría y a pesar de ser un grupo pequeño $(11,8 \%)$, es el grupo de usuarios de Internet que no cuenta con conexión de banda ancha en el hogar el que tiene mayor interés en usar más Internet (63,3\% de respuestas afirmativas). En los otros tres grupos la fracción de quienes quisieran utilizar más Internet es relativamente pareja (entre $34,6 \%$ y $37,8 \%$ ).

Desde el punto de vista de potenciales políticas públicas, resulta interesante analizar en detalle las respuestas del grupo más alejado de Internet (los que ni tienen banda ancha ni son usuarios) respecto a las razones que podrían motivarlos a utilizar Internet. En el Gráfico $\mathrm{N}^{\mathrm{o}} 11$ 


\section{GRÁFICO No 10: PORCENTAJE QUE QUISIERA USAR O USAR MÁS INTERNET, SEGÚN CONDICIÓN DE USUARIO Y TENENCIA DE BANDA ANCHA (2009) (En porcentajes)}

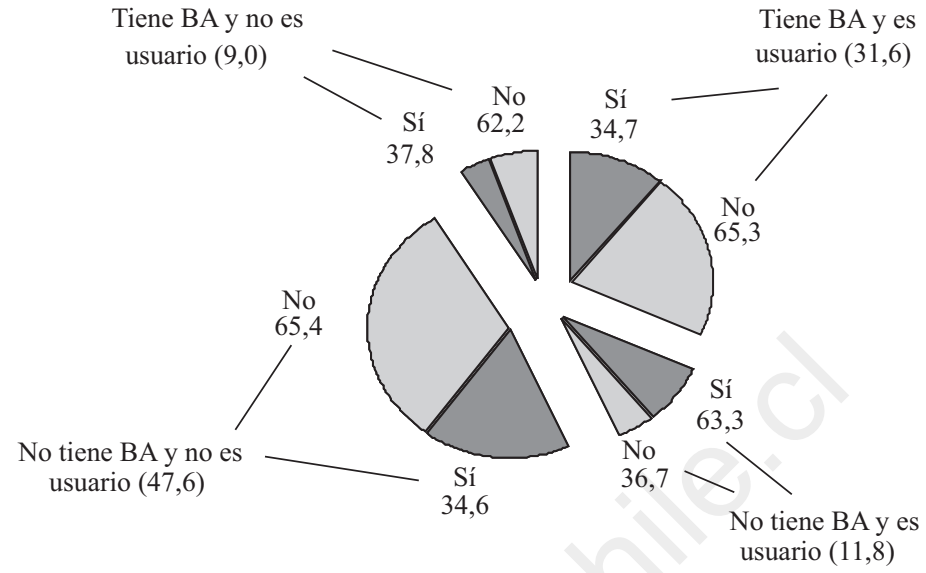

Fuente: Elaboración propia en base a Encuesta sobre Acceso, Uso y Usuarios de Internet Banda Ancha en Chile 2009.

GRÁFICO No 11: RAZONES QUE INDUCIRÍAN A UTILIZAR INTERNET. JEFES DE HOGAR NO USUARIOS SIN CONEXIÓN EN EL HOGAR (2009) (\%)

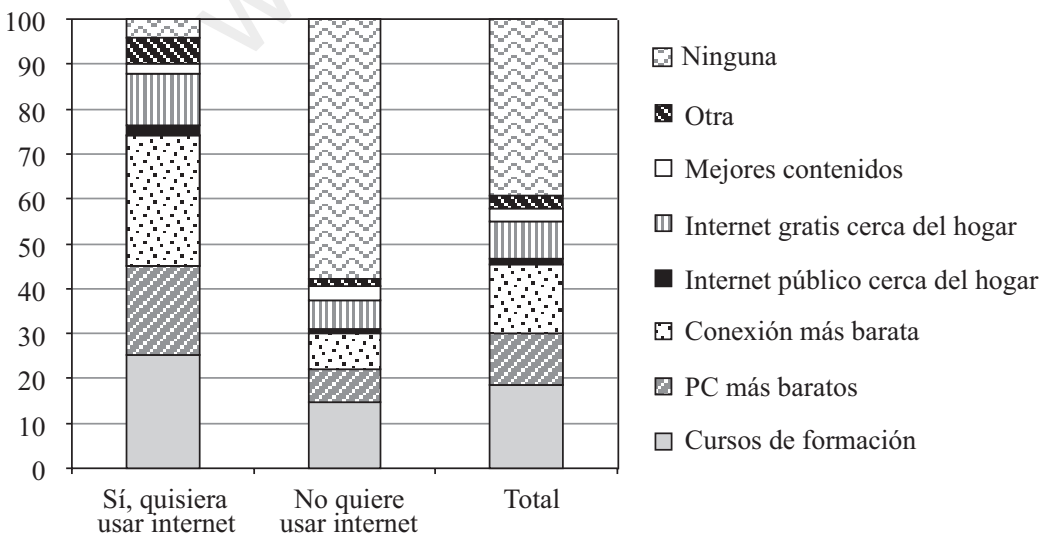

Fuente: Elaboración propia en base a Encuesta sobre Acceso, Uso y Usuarios de Internet Banda Ancha en Chile 2009. 
se resumen las respuestas de este grupo tanto de los que respondieron que sí querrían utilizar Internet como de los que dijeron que no.

Dos elementos destacan del Gráfico $\mathrm{N}^{\mathrm{o}} 11$ : en primer lugar, la importancia relativa de la respuesta "Ninguna" para el grupo que no quiere utilizar Internet. Casi el $60 \%$ de quienes son no usuarios y no tienen banda ancha en la casa simplemente no quieren ser usuarios. En segundo lugar, para el grupo que sí está interesado en usar Internet, el principal freno pareciera ser el precio de la conexión (casi 30\%) y, en segundo lugar, la falta de cursos de formación (25\%) y el costo de los PC (20\%).

El Gráfico $\mathrm{N}^{\circ} 12$ ilustra las respuestas para un segundo grupo que podría ser objetivo de la política pública, el de quienes no tienen conexión de banda ancha y sí son usuarios de Internet. Claramente, éste debiera ser un grupo particularmente receptivo de políticas públicas que pudieran promover el acceso.

Para este grupo, especialmente para quienes declaran que quisieran utilizar más Internet, la razón más preponderante que los llevaría a utilizar más Internet es, de nuevo, el precio de la conexión (alrededor del $30 \%)$. Otro factor relevante es el precio del computador (18\%) y un tercer elemento, claramente menos importante para el grupo de usuarios

GRÁFICO N ${ }^{\circ}$ 12: RAZONES QUE INDUCIRÍAN A UTILIZAR INTERNET.

JEFES DE HOGAR USUARIOS SIN CONEXIÓN EN EL HOGAR (2009) (\%)

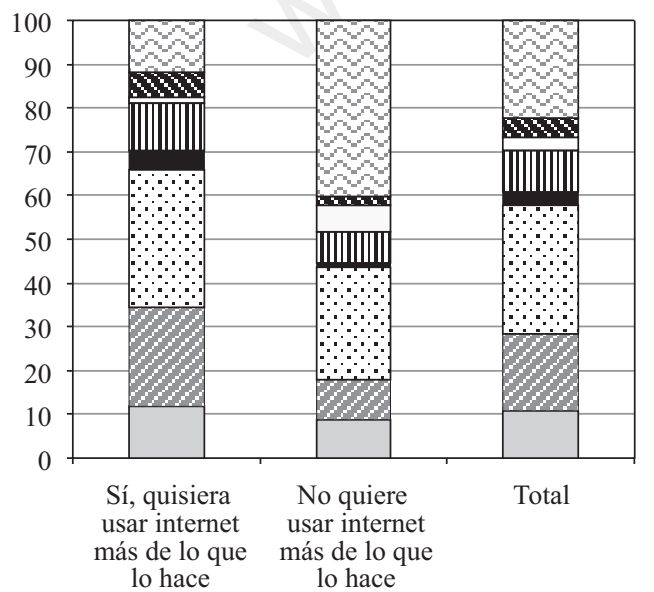
目 Ninguna
Otra
$\square$ Mejores contenidos
$\square$ Internet gratis cerca del hogar
- Internet público cerca del hogar
[.] Conexión más barata
曰 PC más baratos
$\square$ Cursos de formación

Fuente: Elaboración propia en base a Encuesta sobre Acceso, Uso y Usuarios de Internet Banda Ancha en Chile 2009. 
que para los no usuarios, son los cursos de capacitación (11\%). Similar importancia tiene para este grupo la posibilidad de acceder en lugares gratuitos (10\%).

\section{Conclusiones generales}

Este trabajo se ha propuesto caracterizar la "brecha digital" en Chile a partir de los datos de la "Encuesta sobre Acceso, Uso y Usuarios de Internet Banda Ancha en Chile", realizada por el OSUAH, en sus diversas dimensiones que van más allá del acceso en el hogar a banda ancha (e.g., diferencias en uso/no uso de Internet, frecuencias de usos y tipos de usos); también analizar la evolución de esta brecha en aquellas dimensiones que la información disponible en CASEN lo permite (acceso en el hogar y uso/no uso), y analizar a partir de respuestas directas de los encuestados las razones detrás de sus decisiones de suscripción a un servicio de banda ancha y de uso de Internet en general.

$\mathrm{Al}$ respecto, los hallazgos principales del trabajo pueden resumirse así:

- $\quad$ Entre 2006 y 2009 ha habido un crecimiento importante de la banda ancha en Chile. La proporción de hogares conectados creció de $18 \%$ en 2006 a $41 \%$ en 2009 . Este crecimiento de Internet no ha sido exclusivo de ningún grupo en particular (quintil de ingreso, ciudad o nivel educativo), pero sí se observa un mayor crecimiento absoluto en los sectores de mayores ingresos (quintiles 3,4 y 5 en particular) y en el grupo con educación superior (completa o incompleta). El patrón de crecimiento observado ha significado que las brechas absolutas entre los grupos de mayores ingresos y el quintil más pobre se han ampliado. En términos relativos, sin embargo, todas las brechas se han reducido, lo que es natural dados los bajos niveles de penetración de Internet en los estratos de menores ingresos en 2006.

- $\quad \mathrm{Al}$ analizar la evolución de la tasa de uso de Internet entre los jefes de hogar — más allá de que tengan o no conexión— se observa un patrón similar al descrito aunque claramente es superior el aumento en la tasa de uso observado en los quintiles 3 y 4 y, en menor medida, en el segundo quintil.

- $\quad \mathrm{Al}$ analizar las tasas de uso en los diferentes quintiles de ingreso por parte de jefes de hogar que tienen conexión de banda ancha 
en el hogar se encuentra que existe una brecha relacionada con el ingreso: la tasa de uso es mayor en los quintiles de ingresos superiores incluso cuando se compara sólo entre hogares con conexión. Esta brecha, sin embargo, se ha reducido entre 2006 y 2009.

- $\quad$ Respecto a los lugares de conexión se observa una disparidad esperable entre los grupos: los de ingresos superiores hacen uso principalmente en sus hogares y lugares de trabajo, en tanto que los quintiles de menores ingresos se conectan con mayor frecuencia en centros de Internet pagados. La importancia de centros de Internet gratuitos es menor.

- $\quad \mathrm{Al}$ analizar los diferentes usos que los distintos quintiles dan a Internet se observa que: no hay diferencias significativas en lo que hace al uso recreacional; existe una diferencia en cuanto al uso del correo electrónico y la voz sobre Internet (más frecuente en sectores de ingresos altos), pero no de otros servicios de comunicación (e.g., chateo y participación en redes sociales); y hay diferencias en el uso para transacciones económicas y bancarias y en el acceso a información de periódicos y revistas.

- Respecto a las razones por las que no tienen conexión de banda ancha, los jefes de hogar indican como principales motivos el no tener PC (que a su vez está asociado a su costo) y el costo de la conexión de banda ancha. Una tercera razón también relevante es el desinterés y/o desconocimiento del servicio. Esta razón es mencionada como la principal por el $19 \%$ y como la segunda más importante por otro $20 \%$. No se observan diferencias importantes en esta dimensión entre los diversos quintiles.

- $\quad$ Finalmente, respecto a las razones que podrían animar a los no usuarios a utilizar Internet, los jefes de hogar mencionan cursos de formación en primer lugar (15\%) y PC o conexiones más baratos (7,5\% cada uno). Sin embargo, entre los jefes de hogar no usuarios la respuesta más frecuente (casi $60 \%$ ) es "Ninguna". Es decir que, en su mayoría, los no usuarios no tienen interés en serlo.

Consideramos que esta "radiografía" de la brecha digital en Chile es un primer elemento imprescindible para el diseño de cualquier política que busque "fomentar" la banda ancha de alguna manera. El motivo es que en la discusión pública se ha considerado la existencia de esta brecha como razón suficiente para promover una intervención 
pública que aumente fuertemente la penetración de la banda ancha en Chile. Sin embargo, tal decisión requiere analizar desde una perspectiva microeconómica la verdadera magnitud de las fallas de mercado asociadas a la banda ancha (e.g., externalidades de red), desde una perspectiva macroeconómica su potencial impacto en el crecimiento económico y, desde una perspectiva de equidad (y en definitiva política), si el "tamaño" de la brecha amerita la intervención y/o el tiempo que le tomaría "al mercado" reducirla es excesivo ${ }^{11}$. Un buen análisis económico de la magnitud de las externalidades asociadas a la banda ancha y la velocidad a la cual el mercado cerraría las brechas debe ser un requisito previo para considerar distintas alternativas de intervención pública.

Lógicamente, en cualquier caso corresponde realizar un análisis costo/beneficio de las posibles políticas públicas para aumentar la penetración de la banda ancha, lo que requeriría analizar la efectividad de las distintas opciones ${ }^{12}$. Es en este punto que el trabajo contribuye ilustrando distintas dimensiones de la brecha digital que hasta ahora no han sido consideradas en el análisis de políticas públicas. Las propuestas de políticas planteadas hasta ahora se basan en una dimensión de la brecha y a través de un subsidio a la demanda intentan promover el acceso de banda ancha en el hogar. Sin embargo, una política de ese tipo podría resultar inapropiada y poco efectiva al ignorar otros aspectos relevantes como el desconocimiento de algunos grupos de la utilización de la tecnología, el desinterés de otros (posiblemente ligado a un tema de contenidos) o algo tan obvio como el no tener un computador personal. En ese sentido, hay políticas que a la luz de la radiografía de la brecha digital presentada en este trabajo podrían ser más efectivas, como subsidiar la demanda por computadores, capacitar en el uso de computadores e Internet e informar respecto a los usos posibles de Internet.

${ }^{11}$ Ono y Zavodny (2003) encuentran evidencia de que hubo una brecha en el acceso a Internet entre hombres y mujeres durante los 90, la cual desapareció ya en el 2000 sin que existiera ninguna política pública o intervención para reducirla.

${ }^{12} \mathrm{Si}$ bien en otro contexto, un buen ejemplo a considerar respecto al rol que puede jugar la información es el caso de Escocia. Dependiendo de la región, entre 35 y $59 \%$ de las empresas en Escocia creían que no había disponibilidad de banda ancha en su zona geográfica cuando en realidad sí la había. Una campaña de publicidad del gobierno informando beneficios del uso de banda ancha y las regiones en que estaba disponible fue suficiente para aumentar su adopción (Tookey, Whalley y Howick, 2006). Hay otras posibilidades que no es posible analizar con los datos disponibles, como aumentar la enseñanza del inglés en Chile, ya que muchos de los contenidos disponibles en Internet están en inglés y la barrera del lenguaje tiene efectos significativos en reducir la adopción de banda ancha (Prieger y Hu, 2008). 


\section{REFERENCIAS}

Aizu, I. (2002). "A Comparative Study of Broadband in Asia: Deployment and Policy". Asia Network research Discussion Paper, 29 de septiembre.

Autor, D., F. Levy y R. Murnane (2003). "The Skill Content of Recent Technological Change: An Empirical Exploration”. Quarterly Journal of Economics 118(4).

Cava-Ferruela, I. y A. Alabau-Muñoz (2006). "Broadband Policy Assesment: A CrossNational Empirical Analysis". Telecommunications Policy 30.

Chouadrie, J. y Y. Dwivedi (2006). "Investigating Factors Influencing Adoption of Broadband in the Household”. Journal of Computer Information Systems 46(4).

Czernich, N., O. Falck, T. Kretschmer y L. Woessman (2009). "Broadband Infrastructure and Economic Growth". CESifo Working Paper No 2861.

DiMaggio, P., E. Hargittai, C. Celeste y S. Shafer (2004). "Digital Inequality: From Unequal Access to Differentiated Use". En K. M. Neckerman (ed.), Social Inequality. New York: Russell Sage Foundation.

Gillet, S. E., W. H. Lehr y C. A. Osorio (2004). "Local Government Broadband Initiatives". Telecommunications Policy 28.

Gillet, S. E., W. H. Lehr, C., A. Osorio y M. A Sirbu (2006). "Measuring Broadband's Economic Impact". Informe preparado para Economic Development Administration, U.S. Department of Commerce, 28 de febrero.

Hauge, J. y J. E. Prieger (2009). "Demand-Side Programs to Stimulate Adoption of Broadband: What Works?". Working Paper disponible en http://ssrn.com/ abstract $=1492342$.

Howick, S. y J. Whalley (2008). "Understanding the Drivers of Broadband Adoption: The Case of Rural and Remote Scotland". Journal of the Operational Research Society 59 .

Lee, S. y J. Brown (2008). "Examining Broadband Adoption Factors: An Empirical Analysis between Countries". Info 10(1).

Ono, H. y M. Zovodny (2003). "Gender and Internet”. Social Science Quarterly 84(1). (2007). "Digital Inequality: A Five Country Comparison Using Microdata". Social Science Research 36(3).

Prieger, P. E. y W. M. Hu (2008). "The Broadband Digital Divide and the Nexus of Race, Competition, and Quality". Information Economics and Policy 20.

Tookey, A., J. Whalley y S. Howick (2006). "Broadband Diffusion in Remote and Rural Scotland". Telecommunications Policy 28.

Recibido: mayo de 2010. Aceptado: julio de 2010. 